J Clin Pathol 1989;42:483-488

\title{
Granulocytic sarcoma of the cervix: an immunohistochemical, histochemical, and ultrastructural study
}

\author{
S BANIK, A BORG GRECH, B P EYDEN* \\ From the Departments of Histopathology, Royal Infirmary, Blackburn, and the *Christie Hospital and Holt \\ Radium Institute, Manchester
}

SUMMARY Light microscopical and routine immunohistochemical studies of a cervical neoplasm in a 32 year old woman initially suggested a histiocytic lymphoma, but histochemical staining for chloroacetate esterase established the correct diagnosis. This was supported by electron microscopic findings. Eight months later the patient developed a granulocytic sarcoma in her left breast and haemotological features of acute myeloid leukaemia. Accurate intitial diagnosis of granulocytic sarcoma in a non-leukaemic patient may reduce the risk of subsequent acute myeloid leukaemia if appropriate chemotherapy is begun in time.

In the absence of myeloproliferative disease granulocytic sarcoma presents a diagnostic challenge to histopathologists. Regarded as a neoplasm of primitive granulopoietic cells at extramedullary sites, this lesion occurs in young people and most commonly affects soft tissue, bone, periosteum, lymph nodes and skin among other organs. ${ }^{\prime}$ It is rare in the female genital tract, especially the cervix..$^{2-4}$ The lesion usually occurs with concomitant acute myeloid leukaemia or blastic phase of chronic myeloid leukaemia, but may precede the myeloproliferative disease by several months or years. Rarely, the lesion may evolve in myelofibrosis ${ }^{5}$ or polycythaemia vera. ${ }^{6}$ Histological distinction from a histiocytic lymphoma or a high grade non-Hodgkin's lymphoma is difficult and cases recorded as reticulum cell sarcoma terminating in acute myeloid leukaemia have actually been misdiagnosed granulocytic sarcoma.?

\section{Case report}

A 32 year old caucasian woman presented in November 1986 with post-coital bleeding following childbirth two months earlier. In January 1987 a colposcopic examination showed ectropion of the cervix and a punch biopsy specimen was reported to show warty atypia of the squamous epithelium and severe inflammation. The patient had intermittent vaginal bleeding and in October 1987 a large cervical tumour was found. A wedge biopsy specimen of the cervix was obtained and a granulocytic sarcoma of the cervix was diagnosed. At this time a computed tomogram and an ultrasonic scan of the pelvis showed a cervical tumour $(5.5 \mathrm{~cm})$, extending postero-inferiorly into the upper vagina. There was no retroperitoneal or pelvic lymphadenopathy on the scans. The lung fields and mediastinum were normal.

Haematological investigations showed a normal blood picture and a bone marrow examination showed active erythropoiesis with mild megaloid changes. Granulopoiesis showed a normal maturation with 2\% blast cells, but the features were felt to be insufficient to diagnose myelodysplasia. A peripheral blood chromosome analysis did not show any $\mathrm{Ph}^{1}$ chromosome or any other chromosomal aberration. She was treated with pelvic irradiation following which she remained well. A pelvic examination did not show any residual tumour. Eight months after the diagnosis of granulocytic sarcoma she developed a palpable mass in her left breast. An excisional biopsy specimen showed a granulocytic sarcoma of the breast and examination of her peripheral blood and bone marrow showed the features of an acute myeloid leukaemia. She was referred to a regional oncology centre where she was found to have developed further masses in her breasts, a mass in her fauces, and a rightsided proptosis. She received chemotherapy for acute myeloid leukaemia and at the time of writing was in remission. 


\section{Material and methods}

\section{LIGHT MICROSCOPY}

The cervical wedge biopsy specimen measured $2.5 \times 1.0 \times 1.0 \mathrm{~cm}$ and was greyish brown in colour. The breast biopsy specimen consisted of a mass of fibrofatty tissue $(5.5 \times 4.5 \times 4.5 \mathrm{~cm})$ which contained a hard greenish yellow tumour $(4.0 \times 3.0 \times 3.0 \mathrm{~cm})$. Both biopsy specimens were fixed in formalin, embedded in paraffin wax, and 6 micron sections were stained with haematoxylin and eosin.

\section{IMMUNOHISTOCHEMISTR Y}

Immunohistochemical studies were performed on 6 micron formalin fixed, paraffin wax sections using the standard avidin-biotin method and monoclonal antisera for leucocyte common antigen (LCA/CD45), T cell marker (UCHL 1/CD45R), B cell marker (4KB5/ CD45R), epithelial membrane antigen (EMA), cytokeratins (CAM 5.2 and CK1) and granulocyte associated antigen (anti-M-1/CD15). All these reagents were obtained from Dakopatts except cytokeratin CAM 5.2 which was from Becton-Dickinson. Polyclonal antisera were used for muramidase (lysozyme, Dakopatts), $\alpha 1$-antitrypsin (AAT, Dakopatts), cathepsin $G$, and leucocyte elastase. The last two antisera were kindly provided by Dr J Crocker. Suitable positive and negative controls were used.

\section{HISTOCHEMISTRY}

A stain for naphthol-ASD-chloroacetate esterase (CAE) was performed on the paraffin wax section using Leder's technique. ${ }^{8}$

\section{ELECTRON MICROSCOPY}

Small blocks of tissue from the cervical neoplasm were retrieved from formalin and conventionally processed into Agar 100 (Epon-type) epoxy resin. Ultrathin sections were stained with $5 \%$ aqueous uranyl acetate and lead citrate and examined in anAE1 801 electron microscope.

\section{Results}

\section{LIGHT MICROSCOPY}

A review of the original colposcopic biopsy specimen showed reactive atypia of the squamous epithelium. The subepithelial zone contained infiltrates of lymphocytes, plasma cells, and neutrophils but the main bulk of the cervical stroma contained a monomorphic infiltrate of neoplastic cells which were similar to that of the subsequent biopsy specimens.

The wedge biopsy specimen of the cervix showed neoplastic cells in its stroma, sparing the endocervical crypts, and blood vessels with ulceration of the mucosa. Similar neoplastic infiltrates were present in endometrial curettings and in a lateral vaginal wall biopsy specimen.

The neoplasm consisted of diffuse sheets of large cells with round non-cleaved nuclei and scanty indistinct amphophilic cytoplasm (fig 1). Some of the cells contained inconspicuous central nucleoli. There was a high incidence of bizarre mitoses. Some areas showed a fasciculated morphology with sclerosis or fibrosis of the interstitium. "Starry sky" appearances or tingible body macrophages were not present. Scanty eosinophilic leucocytes were interspersed among the tumour cells. The histological appearances of the breast neoplasm were similar to those of the cervical lesion. A few scattered eosinophilic myelocytes were also present.

\section{IMMUNOHISTOCHEMISTR Y}

The stain for leucocyte common antigen was positive only in the inflammatory cells on the surface but not within the neoplastic cells. The tumour cells were also negative for T and B cell markers, CAM 5.2, CK1 and EMA. There was a strong positivity for lysozyme, AAT (fig 2a), and anti-M-1 in most tumour cells. Similar positive staining was seen in the stains for cathepsin G and leucocyte elastase.

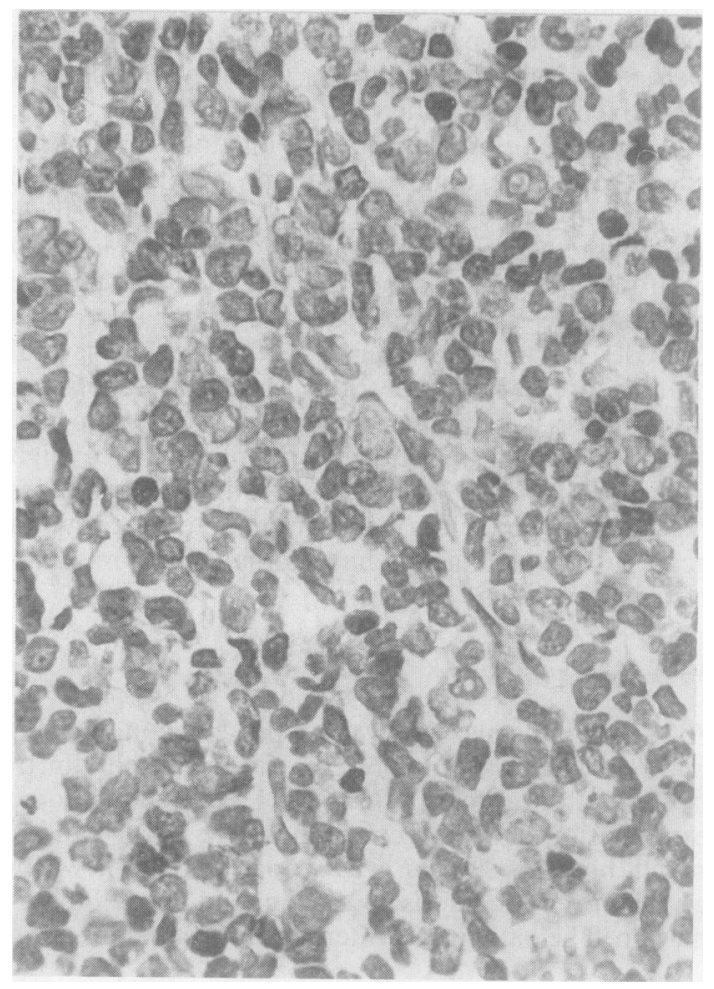

Fig 1 Cytomorphology of the granulocytic sarcoma of the cervix. (Haematoxylin and eosin.) 


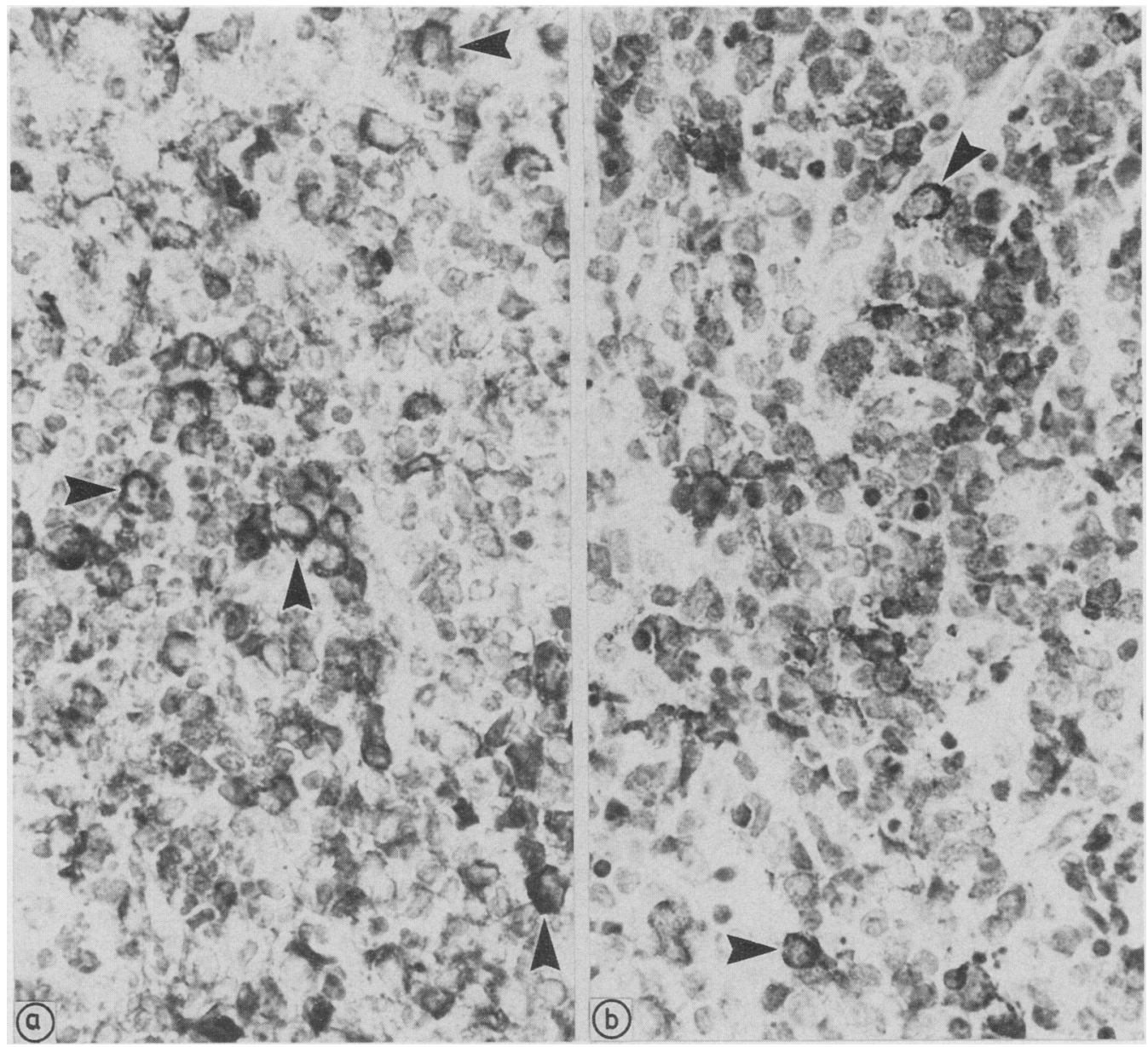

Fig 2 (a) Immunostaining for $\alpha$-1-antitrypsin and (b) histochemical staining for chloroacetate esterase. Arrowheads mark cells with particularly intense staining.

\section{HISTOCHEMISTRY}

The neoplastic cells showed a strongly positive staining reaction for chloroacetate esterase (fig $2 b$ ).

\section{ELECTRON MICROSCOPY}

Ultrastructurally, the cervical specimen contained reactive elements but the bulk consisted of neoplastic myeloid cells in varying degrees of maturation (fig 3 ). A few were myeloblasts with a rounded multinucleolate euchromatic nucleus and a narrow cytoplasmic rim containing few or no granules. The remaining cells showed varying degrees of nuclear irregularity, nucleolar development, peripheral heterochromatin and cytoplasmic granularity consistent with promyelocytes, and granulocytes of intermediate and late type. ${ }^{9}$ Many of the granules were acceptable as of primary (azurophilic) type (fig 4), although others seemed to have a partially extracted content.

\section{Discussion}

Diagnostic distinction from high grade nonHodgkin's lymphoma, especially histiocytic lymphoma, is difficult when granulocytic sarcoma appears at extramedullary sites in a non-leukaemic patient. Thus in a large series of 61 biopsied cases of granulocytic sarcoma the correct diagnosis was made or suspected in only $44 \%$ of cases. ${ }^{1}$ The presence of eosinophilic myelocytes provides an important clue towards the diagnosis, but these cells are present in variable numbers and only in those lesions which exhibit a maximal degree of differentiation. Poorly differentiated granulocytic sarcomas or lesions in the 


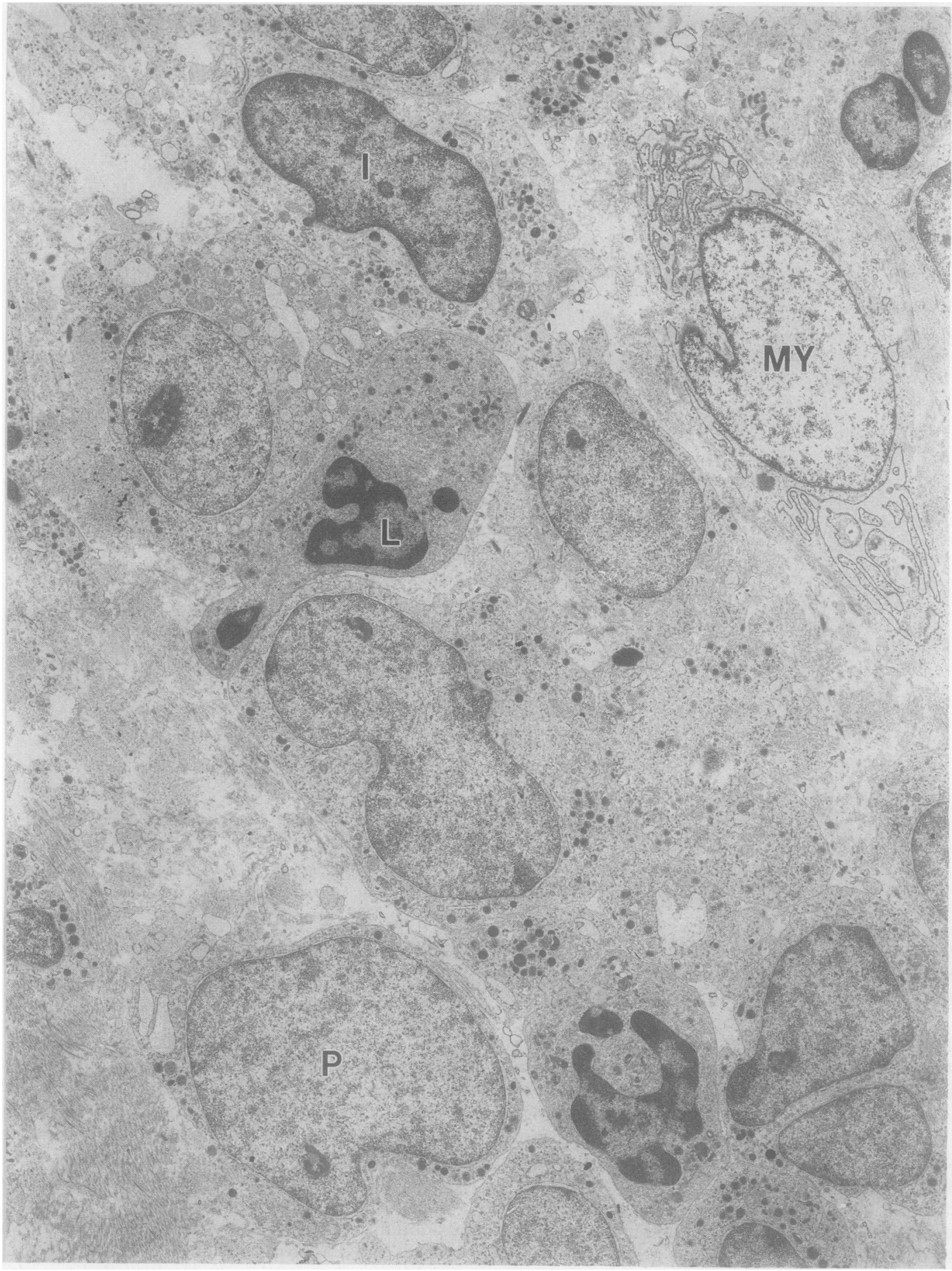

Fig 3 Electron micrograph of the cervical biopsy specimen showing varied nuclear appearances. $P$, early promyelocyte; I, intermediate granulocyte; $L$, late stage granulocyte; $M Y$, myofibroblast. 


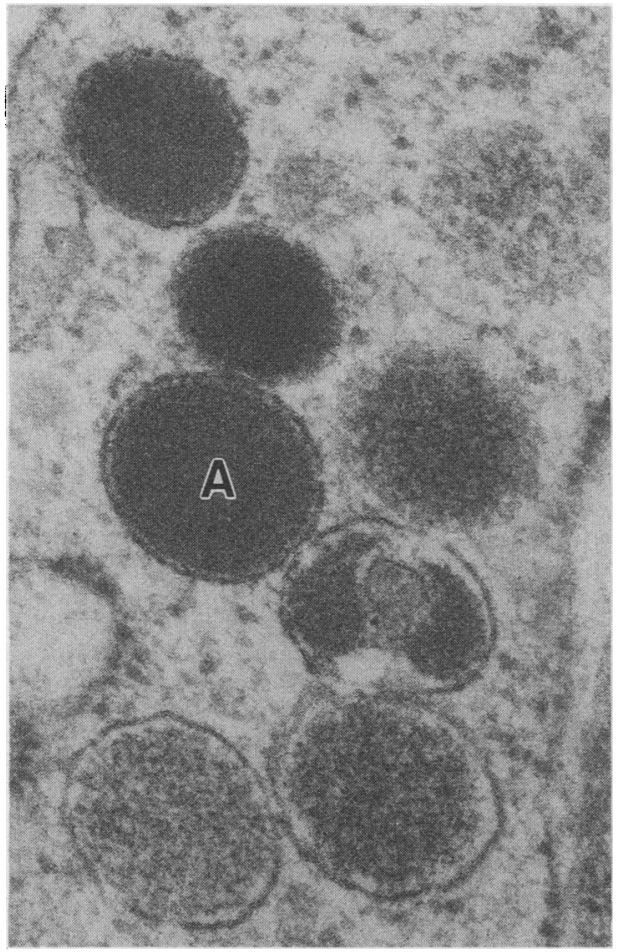

Fig 4 Electron micrograph showing detail of a promyelocyte from the cervical biopsy specimen. A, typical primary or azurophilic granule; other granules have irregular or partially extracted contents.

blastic phase contain hardly any eosinphilic leucocytes at all, thereby rendering the diagnosis difficult. On the other hand, the presence of eosinophilic myelocytes in a case of $T$ cell lymphoma has led to an erroneous initial diagnosis of granulocytic sarcoma. ${ }^{10}$

The role of histochemical and immunohistochemical studies in the diagnosis of granulocytic sarcoma cannot be overemphasised. ${ }^{11-15}$ Polyclonal antisera against muramidase (lysozyme), AAT, leucocyte elastase and cathepsin $G$ show reactivity against granulocytes. ${ }^{14}$ Of these, anti-lysozyme and anti-AAT stain cells of the granulocytic series from promyelocytes onwards, although overlapping occurs with histiocytes, monocytes, and some epithelial cells. Leucocyte elastase and cathepsin G have been shown to be effective granulocytic markers in the diagnosis of granulocytic sarcoma. ${ }^{16}$

As histiocytic lymphomas show variable positivity for lysozyme and AAT a granulocytic sarcoma can be easily misdiagnosed as histiocytic lymphoma unless a specific granulocytic marker is used. Furthermore, granulocytic sarcoma is rare, in contrast to the relative frequency of histiocytic lymphomas. The distinction between these two tumours was achieved with the help of CAE. Most reports have emphasised the usefulness of the CAE stain in the diagnosis of granulocytic sarcoma. ${ }^{2} 151718$ In the series of Neiman et al 42 of 57 cases $(75 \%)$ stained positively for CAE. In 10 of the 15 negative cases the histological material was fixed in Zenker's fluid, indicating that fixation is critical for optimal staining. In the same series 42 of 47 cases $(89 \%)$ stained positively for lysozyme.

The ultrastructural findings provide additional support for the diagnosis of granulocytic sarcoma because the combination of nuclear features with an appropriate degree of cytoplasmic granulation is instrumental in defining granulocytic differentiation. ${ }^{9}$ The spectrum of appearances-at one end a sparse granule-free cytoplasm enclosing a regularly shaped, multinucleolate euchromatic nucleus, while at the other an abundant and prominently granular cytoplasm surrounding a deeply dissected anucleolate nucleus with abundant heterochromatin-is compatible only with neutrophilic differentiation. No lymphoma approaches this spectrum of nuclear appearances, although in true histiocytic lymphoma nuclei may also vary from being rounded and regularly shaped to lobated, segmented, or highly irregular. ${ }^{19} 20$ In these lymphomas, however, both the irregular and rounded nuclei have prominent ${ }^{20}$ and often multiple ${ }^{19}$ nucleoli, as well as finely dispersed chromatin. This is the reverse of the stituation in granulocytic differentiation where multiple nucleoli and a euchromatic picture correlate exclusively with undistorted nuclear profiles, as typified by the myeloblast.

The smoothly contoured membrane and the homogenously dense contents of the granules in this tumour are typical features of primary lysosomes which have been documented from a wide variety of cells. ${ }^{21}$ Their presence in numbers excludes most lymphomas from diagnostic consideration and while they occur in true histiocytic lymphoma, these tumours also have a conspicuous development of large phagolysosomes and residual bodies with a dense and heterogenous content. ${ }^{1920}$ These secondary lysosomes are not found to the same extent in granulocytic tumours.

The accuracy of histological diagnosis is crucial because of the disparate biological behaviour of granulocytic sarcoma and histiocytic lymphoma. Furthermore, the prognosis and treatment of these two lesions are dissimilar. Thirteen of 15 cases $(87 \%)$ of granulocytic sarcoma without a concomitant leukaemia underwent transformation into acute myeloid leukaemia, the time interval varying from one to 49 months in the series of Neiman et al. ${ }^{1}$ Cases have been recorded, however, in which such a sequential transformation did not take place. ${ }^{12} 172223$ Chemotherapy in the same regimen as that used for treating acute myeloid leukaemia prevented the progression into 
leukaemia in these cases, although it was not stated whether the four cases were treated as acute leukaemia. ${ }^{23}$ It has been suggested that treatment regimens for acute myeloid leukaemia should be given in cases of non-leukaemic granulocytic sarcoma to prevent transformation into acute leukaemia, and bone marrow transplantation should be considered during clinical remission. ${ }^{15}$ If such a therapeutic regimen proves effective it will further the importance of accurately diagnosing granulocytic sarcoma.

We are indebted to Dr David Deakin, Christie Hospital and Holt Radium Institute for kindly providing the clinical data, Dr John Crocker, East Birmingham Hospital for the kind supply of cathepsin $G$ and leucocyte elastase antisera, and Mr David Rushworth for technical assistance.

\section{References}

1 Neiman RS, Barcos M, Berard C, et al. Granulocytic sarcoma: a clinicopathologic study of 61 biopsied cases. Cancer 1981;48:1426-37.

2 Seo IS, Hull MT, Pak HY. Granulocytic sarcoma of the cervix as a primary manifestation; case without overt leukaemic features for 26 months. Cancer 1977;40:3030-7.

3 Steinbock GS, Morrisseau PM, Vinson RK. Acute obstructive renal failure secondary to granulocytic sarcoma (chloroma). Urology 1986;27:268-70.

4 Chorlton I. Malignant lymphoma of the female genital tract and ovaries. In: Fox H, ed. Obstetrical and gynaecological pathology. Edinburgh: Churchill Livingstone, 1987:758.

5 Jacobs P, Sellars S. Granulocytic sarcoma preceding leukaemic transformation in myelofibrosis. Postgrad Med J 1985;61: 1069-71.

6 MacCullum PK, Newbould MJ, Sambrook PS, Burton IE. Extramedullary haemopoietic tumours complicating polycythaemia vera. J Clin Pathol 1988;41:609-14.

7 Wiernik PH, Serpick AA. Granulocytic sarcoma (chloroma). Blood 1970;35:361-9.

8 Latham GR. Routine laboratory methods. In: Stansfeld AG, ed. Lymph node biospy interpretation. Edinburgh: Churchill Livingstone, 1985:35.
9 Azar HA. The haematopoietic system. In: Trump BF, Jones RT, ed. Diagnostic electron microscopy. Vol 2. New York: John Wiley \& Sons, 1979:47-161.

10 Whitcomb CC, Sternheim WL, Borowitz MJ, Davila E, Byrne GE. T cell lymphoma mimicking granulocytic sarcoma. Am J Clin Pathol 1985;84:760-3.

11 Muss HM, Moloney WC. Chloroma and myeloblastic tumours. Blood 1973;42:721-8.

12 McCarty KS Jr, Wortman J, Daly J, Rundles RW, Hanker JS. Chloroma (granulocytic sarcoma) without evidence of leukaemia: facilitated light microscopic diagnosis. Blood 1980;56:104-8.

13 Yam LT. Granulocytic sarcoma with pleural involvement: identification of neoplastic cells with cytochemistry. Acta Cytol 1985;29:63-6.

14 Crocker J, Burnett D. Granulocytic markers in histopathology: a review. J Pathol 1986;150:77-88.

15 Mansi JL, Selby PJ, Carter RL, Powles RL, McElwain TJ. Granulocytic sarcoma: a diagnosis to be considered in usual lymphoma syndromes. Postgrad Med J 1987;63:447-9.

16 Muller S, Sangster G, Crocker J, et al. An immunohistochemical and clinico-pathological study of granulocytic sarcoma (chloroma). Haematol Oncol 1986;4:101-12.

17 Beck TM, Day JC, Smith CE, Eddy HE. Granulocytic sarcoma treated as an acute leukaemia-report of a case. Cancer 1984;53:1764-6.

18 Callahan M, Wall S, Askin F, Delaney D, Koller C, Orringer EP. Granulocytic sarcoma presenting as pulmonary nodules and lymphadenopathy. Cancer 1987;60:1902-4.

19 Mirchandani ILA, Shah ILA, Palutke M, et al. True histiocytic lymphoma-a report of four cases. Cancer 1983;52:1911-18.

20 Thomas P,Said JW, Rosenfelt FP, Heifetz, LJ. True histiocytic lymphoma: an immunohistochemical and ultrastructural study of two cases. Am J Clin Pathol 1984;81:243-8.

21 Erlandson RA. Diagnostic transmission electron microscopy of human tumours. New York: Masson, 1981:36.

22 Eshghabadi M, Shojania AM, Carr I. Isolated granulocytic sarcoma: report of a case and review of the literature. J Clin Pathol Oncol 1986;4:912-17.

23 Meis JM, Butler JJ, Osborne BM. Granulocytic sarcoma in nonleukaemic patients. Lab Invest 1986;54:42A.

Requests for reprints to: Dr S Banik, Department of Histopathology, Royal Infirmary, Blackburn, BB2 3LR, England. 